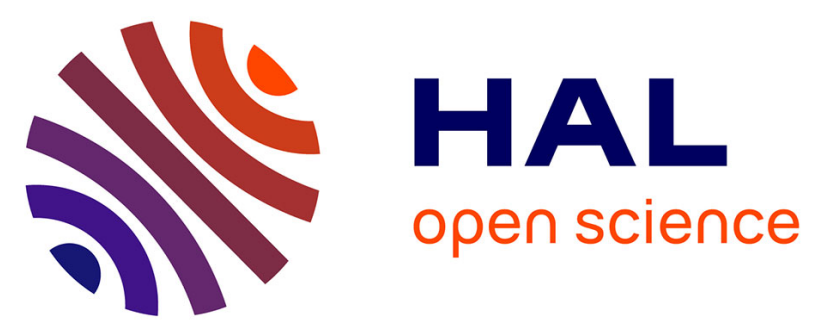

\title{
Comparison between x-ray diffraction and quantitative surface calorimetry based on infrared thermography to evaluate strain-induced crystallinity in natural rubber
}

\author{
Jean-Benoit Le Cam, Pierre-Antoine Albouy, Sylvain Charles
}

\section{To cite this version:}

Jean-Benoit Le Cam, Pierre-Antoine Albouy, Sylvain Charles. Comparison between x-ray diffraction and quantitative surface calorimetry based on infrared thermography to evaluate strain-induced crystallinity in natural rubber. Review of Scientific Instruments, 2020, 91 (4), 10.1063/1.5141851 . hal-02562286v2

\section{HAL Id: hal-02562286}

https://hal-univ-rennes1.archives-ouvertes.fr/hal-02562286v2

Submitted on 15 May 2020

HAL is a multi-disciplinary open access archive for the deposit and dissemination of scientific research documents, whether they are published or not. The documents may come from teaching and research institutions in France or abroad, or from public or private research centers.
L'archive ouverte pluridisciplinaire HAL, est destinée au dépôt et à la diffusion de documents scientifiques de niveau recherche, publiés ou non, émanant des établissements d'enseignement et de recherche français ou étrangers, des laboratoires publics ou privés. 


\title{
Comparison between X-ray diffraction and quantitative surface calorimetry based on infrared thermography to evaluate strain-induced crystallinity in natural rubber
}

\author{
J.-B. Le Cam, ${ }^{1}$ P.-A. Albouy, ${ }^{2}$ and S. Charlès ${ }^{1}$ \\ 1) Université de Rennes, CNRS, Institut de Physique de Rennes, UMR 6251, F-35000 Rennes, France \\ ${ }^{2)}$ Laboratoire de Physique des Solides, CNRS, Université Paris-Sud, Université Paris-Saclay, \\ 91405 Orsay, France
}

The crystallinity of stretched crystallizable rubbers is classically evaluated using X-ray diffraction (XRD). As crystallization is a strongly exothermal phenomenon, quantitative surface calorimetry from infrared thermography (IRT-QSC) offers an interesting alternative to XRD for determining the crystallinity. In the present paper, the two measurement techniques have been used for evaluating of the strain-induced crystallinity of the same unfilled natural rubber. This study provides the first comparison between the two techniques. Results obtained highlight a very satisfactory agreement between the two measurements, which opens a simple way for evaluating the strain-induced crystallinity from temperature measurements.

\section{INTRODUCTION}

Since the pioneering work by Katz in $1925^{1}$, who obtained the first XRD pattern of a stretched natural rubber (NR), the straininduced crystallization (SIC) of rubber is classically investigated using X-ray diffraction (XRD). XRD provides the crystallinity but also information of paramount importance on the crystalline phase structure ${ }^{2,3,4,5}$, chain orientation ${ }^{6}$, kinetics of crystallization $^{7,8}$, non exhaustively.

Concerning the crystallinity measurement, Göritz and coworkers showed in the 1970s that strain-induced crystallinity could be quantified accurately with an alternative technique, based on "stretch calorimetry"9. Indeed, crystallization is strongly exothermal and the corresponding crystallinity can therefore be evaluated from the part of the total heat source ${ }^{1}$ that is produced by SIC only. This technique offered a simpler way than XRD to evaluate the crystallinity. Despite this, calorimetry under stretching was no longer used to measure crystallinity. A possible reason could be that the crystallinity obtained was averaged over all the specimen. Therefore, it is not possible to address heterogeneous crystallinity fields, typically at the crack tip of a notched specimen $^{10,11}$. With the advent of non contact measurement techniques, it has been demonstrated that the calorimetric response could be directly obtained at the surface of a thin specimen as soon as the heat diffusion by conduction and convection is characterized and that no temperature gradient occurs in the specimen thickness $^{12}$. This technique has been successfully employed for characterizing the calorimetric signature of phenomena involved in the rubber deformation under homogeneous strain states ${ }^{13,14}$ and at the crack tip where the mechanical and calorimetric fields are strongly heterogeneous ${ }^{15,16}$. Furthermore, such technique enables us to make energy balance and to identify the intrinsic dissipation $^{17,18,19}$.

Recently, Ref. 20 has proposed to couple the work by Göritz and co-workers and the IR thermography-based surface calorimetry approach to evaluate the strain-induced crystallinity ${ }^{20}$. By this way, the author measured the crystallinity induced in an unfilled natural rubber submitted to a mechanical tensile cycle. Even though the crystallinity found seems to be in good agreement with the literature, no validation was done by comparing the result obtained with that obtained with XRD for the same material. This is the aim of the present study. In the first part of the paper, the

\footnotetext{
${ }^{1}$ The term 'heat source' is used in this paper to mean the heat power density in $\mathrm{W} / \mathrm{m}^{3}$, which is produced or absorbed by the material.
}

framework for evaluating the strain-induced crystallinity from the two techniques is presented. The second part of the paper details the experiments carried out and compares the two measurements performed. Concluding remarks close the paper.

\section{XRD TECHNIQUE}

Crystallinity indices reported here are derived from an analysis of angular scans centered on the amorphous halo as detailed in Ref. $^{21}$. This method combines simplicity and direct access to the Herman orientation parameter for the amorphous phase. Further parameters provided by X-ray diffraction include the crystallite dimensions and their orientation with respect to the draw axis (see the references cited in the introduction section). One main limitation for the use of XRD is that it requires the installation of a testing machine on a laboratory diffraction bench, which is out of the reach of many laboratories. In the present case the testing machine is installed on a rotating anode generator operated at medium power (copper anode, $40 \mathrm{kV}, 40 \mathrm{~mA}$, focus size: $\left.0.2 \times 0.2 \mathrm{~mm}^{2}\right)$. The $C u K_{\alpha}$ radiation is selected with a doubly curved graphite monochromator with a focalization distance of 24 $\mathrm{cm}$. The sample is located at the focalization point which ensures optimized diffracted intensity. The set-up can be equipped with an indirect illumination CCD camera or a hybrid pixel detector. This last X-ray camera combines high efficiency and absence of noise and acquisition times as low as $0.2 \mathrm{~s}$ per frame with good counting statistics are possible ${ }^{22}$. The drawing speed can be selected between $1 \mathrm{~mm} / \mathrm{min}$ and $800 \mathrm{~mm} / \mathrm{min}$. However the necessity of collecting a sufficient number of frames (acquisition time: $0.2 \mathrm{~s}$ ) during the stretching phase limited the maximal drawing speed to $200 \mathrm{~mm} / \mathrm{min}$. The incident beam diameter is ca. $1 \mathrm{~mm}$ in that case, which precludes any detailed analysis of heterogeneous zones if any. Much smaller beam spots can be reached with special optics at the price of intensity loss and systematic mapping becomes highly time consuming.

\section{QUANTITATIVE SURFACE CALORIMETRY FROM IR THERMOGRAPHY (IRT-QSC)}

SIC is a strongly exothermal phenomenon, which explains why surface calorimetry is a relevant alternative to determine the crystallinity. Crystallinity can be evaluated from the part of the total heat source that is produced by SIC only, which gives access to the corresponding crystallization temperature $T_{\text {cryst }}$. The crystallinity $\chi$ can then be deduced from $T_{\text {cryst }}$ by considering that the crystallization energy of natural rubber can be approximated 
by the enthalpy of fusion $\Delta H_{\text {cryst }}$ (in $\left.\mathrm{J} / \mathrm{dm}^{3}\right)^{20,23}$ :

$$
\chi(t)=\frac{\rho C T_{\text {cryst }}(t)}{\Delta H_{\text {cryst }}}
$$

$\rho$ and $C$ are respectively the material's density (in $\mathrm{kg} / \mathrm{dm}^{3}$ ) and the heat capacity (in $\mathrm{J} /(\mathrm{kg} \mathrm{K})$ ). The material's density and heat capacity are assumed to be independent of strain and temperature.

Determining strain-induced crystallinity from infrared (IR) thermography has several advantages:

- the crystallinity field is measured instantaneously, which is of paramount importance in case of heterogeneous crystallinity field;

- IR thermography provides high resolution thermal measurements (temporal, spatial and on the value of the crystallinity itself through the thermal resolution of $20 \mathrm{mK}$ in the temperature range of the present experiment),

- the measurement can be performed in any lab equipped with a conventional testing machine,

- the heat source produced by SIC can be directly linked with constitutive equations through the thermomechanical couplings. Therefore, this technique is all the more interesting that it enables us to validate and to improve thermomechanical SIC models.

Nevertheless, this technique does not provide information on the crystalline phase structure and chain orientation. In the next section, the thermomechanical framework used to determine the heat source and the crystallization temperature due to SIC is presented.

\section{HEAT SOURCE RECONSTRUCTION}

Most of mechanical tests are conducted under non-adiabatic. The temperature measured is therefore affected by heat diffusion within the specimen and with the specimen outside. Therefore, changes in temperature are not only due to the material deformation itself, and the heat diffusion equation is used to determine the corresponding heat source from temperature measurement. This quantity is intrinsic to the material deformation and can be directly compared with constitutive model predictions. Thus, any temperature measurement technique can be used as a calorimeter, an IR camera in the present study, as soon as the heat diffusion by conduction and convection is characterized. In the thermodynamic framework applied, any thermodynamical system out of equilibrium is considered as the sum of several homogeneous subsystems at equilibrium. The deformation is considered as a quasi-static thermodynamic process. The equilibrium state of each volume material element is defined by $n$ state variables: the absolute temperature $T$, the deformation gradient tensor $\mathbf{F}$ and $m(=n-2)$ internal tensorial variables $\boldsymbol{\xi}_{\alpha}$. The local form of the heat diffusion equation writes as follows in the Lagrangian configuration:

$$
\rho C \dot{T}-\operatorname{Div}\left(\boldsymbol{\kappa}_{0} \operatorname{Grad} T\right)=R+\underbrace{\mathscr{D}_{\text {int }}+T \frac{\partial \mathbf{P}}{\partial T}: \dot{\mathbf{F}}+T \sum_{\beta=1}^{m} \frac{\partial \boldsymbol{A}_{\boldsymbol{\beta}}}{\partial T}: \dot{\boldsymbol{\xi}_{\boldsymbol{\beta}}}}_{S}
$$

where $\kappa_{0}$ is a positive semi-definite tensor characterizing the thermal conductivity of the material. $R$ stands for the external heat source due to radiation. $S$ denotes the overall heat source induced by the deformation process. The term $\mathscr{D}_{\text {int }}$ corresponds to the intrinsic dissipation. The term $T \frac{\partial \mathbf{P}}{\partial T}: \dot{\mathbf{F}}$ is the heat source due to couplings between temperature and strain, where $\mathbf{P}$ is the nominal stress tensor. The term $T \frac{\partial A_{\beta}}{\partial T}: \dot{\boldsymbol{\xi}_{\boldsymbol{\beta}}}$ corresponds to the other thermomechanical couplings (for instance related to phase change in the material). Let us denote $\theta$ the temperature variation with respect to the equilibrium temperature $T^{\text {ref }}$ in the reference state, corresponding to the undeformed state ( $T^{\text {ref }}$ is constant and equal to the ambient temperature). In case where changes in ambient temperature occur, $T^{\text {ref }}$ has to be corrected accordingly with the measurement of ambient temperature variations. Moreover, in the case where the heat conduction in the specimen plane is neglected, the tri-dimensional formulation of the heat diffusion equation can be simplified and written in case of homogeneous heat source field. After some calculations that are not detailed here, the heat diffusion equation can be rewritten in case of homogeneous heat source field ${ }^{12}$ :

$$
\rho C\left(\dot{\theta}+\frac{\theta}{\tau}\right)=S
$$

where $\tau$ is a parameter characterizing the heat exchanges between the specimen and its surroundings ${ }^{2}$. It can be easily identified from a natural return to room temperature after a heating (or a cooling) for each testing configuration (machine used, environment, etc). For instance in case where the material is beforehand heated, the exponential formulation of the temperature variation is used to determine parameter $\tau: \theta=\theta_{0} \exp \left(-\left(t-t_{0}\right) / \tau\right)$. In case of large deformations, $\tau$ depends on the stretch. Either $\tau$ is determined at different increasing stretches, further details are provided in Ref. ${ }^{13}$, or the value of $\tau$ is corrected according to its dependency to the stretch in case of incompressible materials. In the former case, the determination of $\tau$ during the natural return to ambient temperature for stretches higher than that at which crystallization starts is affected by the fact that additional heat is produced (absorbed) due to crystallization (melting) during the material cooling (heating). This is the reason why in the present study $\tau(\lambda)$ has been determined from its value in the undeformed state (denoted $\tau_{0}$ ) and the link between the thickness and the stretch. This link depends on the biaxiality ratio $B$, defined as the ratio between the logarithm of $\lambda_{2}$ and the logarithm of $\lambda_{1}$. $\lambda_{1}$ and $\lambda_{2}$ are the maximum and minimum principal stretches in the specimen plane, respectively. By assuming the material to be incompressible, $\tau$ writes:

$$
\tau=\tau(\lambda, B)=\tau_{0} \lambda^{-B-1} .
$$

In the present case of the uniaxial tension, $B$ is equal to -0.5 and $\tau$ writes finally:

$$
\tau(\lambda)=\frac{\tau_{0}}{\sqrt{\lambda}}
$$

\section{A. Determination of the crystallinity}

Figure 1 illustrates the methodology proposed. It requires the temperature variation $\theta$, parameter $\tau$ and the thermophysical parameters $\rho, C$ and $\Delta H$ as input data, and is composed of four steps:

\footnotetext{
${ }^{2}$ In this case, only a one-point temperature measurement is required, meaning that the crystallinity can be evaluated with a pyrometer
} 

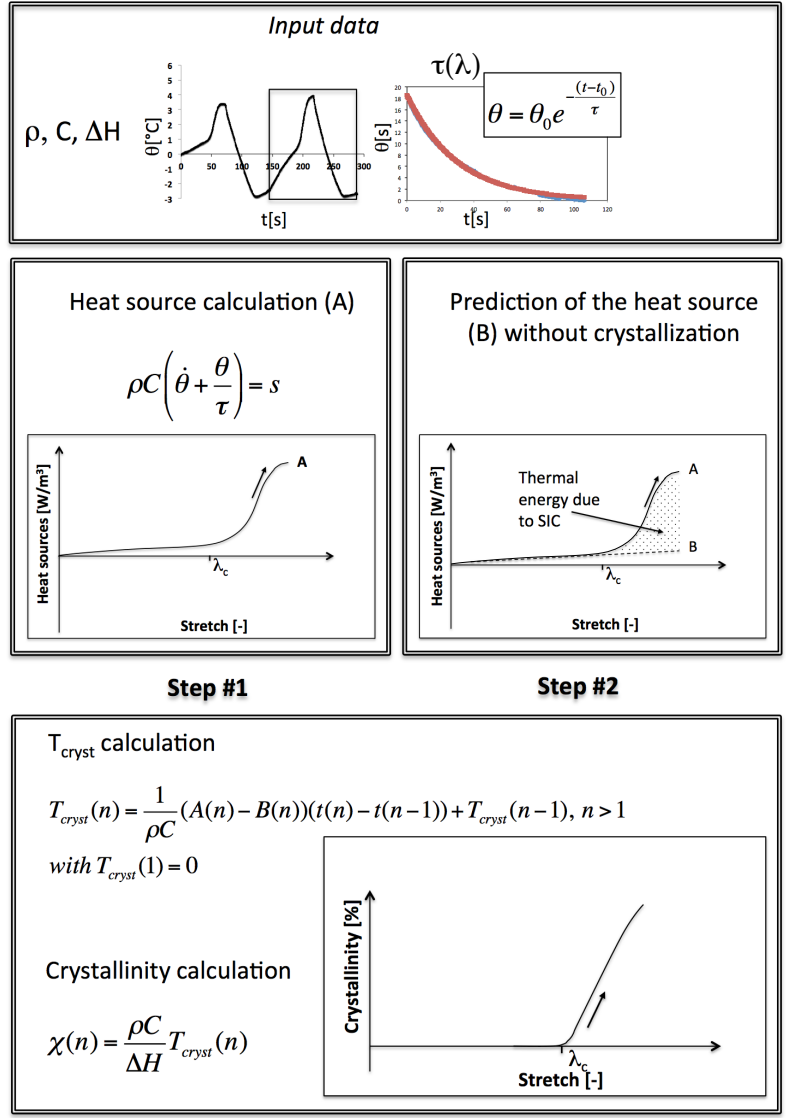

Steps \#3 and \#4

FIG. 1. Methodology for determining crystallinity from temperature variation measurement

Step\#1: The heat source is calculated by applying Equation 3. The diagram illustrates the typical increase in the heat source produced in unfilled NR once crystallization starts $^{9,13}$. Here, $\lambda_{c}$ stands for the stretch at which SIC starts;

Step \#2: The thermal energy due to SIC is deduced from the area located between the heat source measured (curve A) and the part of the heat source that would be due to the elastic coupling only (curve B). The latter is predicted by using a polynomial, whose parameters are identified by fitting the heat source measured before SIC starts $\left(\lambda<\lambda_{c}\right)$ : $C_{1}\left(\lambda-\lambda^{-2}\right)+C_{2}\left(\lambda-\lambda^{-2}\right)^{2}+C_{3}\left(\lambda-\lambda^{-2}\right)^{3}$. It should be noted that a first or second order polynomial also provides satisfactorily results. As the tests are performed under controlled displacement, the strain rate effect is accounted by a constant that is multiplied with the $C_{i}$ parameters;

Step \#3: $T_{\text {cryst }}$ is computed from the heat source due to SIC, i.e. the area between curves A and B. See for instance the not centered numerical scheme given in the figure;

Step \#4: The crystallinity is calculated by applying Equation (1).

This method is very simple and does not require measuring the nominal stress variations nor characterizing possible non-entropic effects $^{24}$, because they are included in the calorimetric response.

\section{EXPERIMENTS}

The material considered here is an unfilled natural rubber of grade SMR 5L vulcanized by sulfur (1.5 phr) in the presence of conventional activators and antioxidant agents. The average molecular weight between cross-links is $6330 \mathrm{~g} \mathrm{~mol}^{-1}$ ( 86 isoprene units) based on mechanical measurements. For the calculations, the density, specific heat and fusion enthalpy values for the calculations were chosen equal to $0.936 \mathrm{~kg} / \mathrm{dm}^{3}, 1768 \mathrm{~J} /(\mathrm{kg} \mathrm{K})$ and $62 \times 10^{3} \mathrm{~J} / \mathrm{dm}^{3}$, respectively. The mechanical loading is applied symmetrically at a stretch equal to 7.2 and two different loading rates: 100 and $200 \mathrm{~mm} / \mathrm{min}$. The averaged specimen dimensions were $19.3 \mathrm{~mm}$ in height, $7.2 \mathrm{~mm}$ in width and 1.4 in thickness. The experimental set-up used for the XRD technique is depicted in Figure 2.

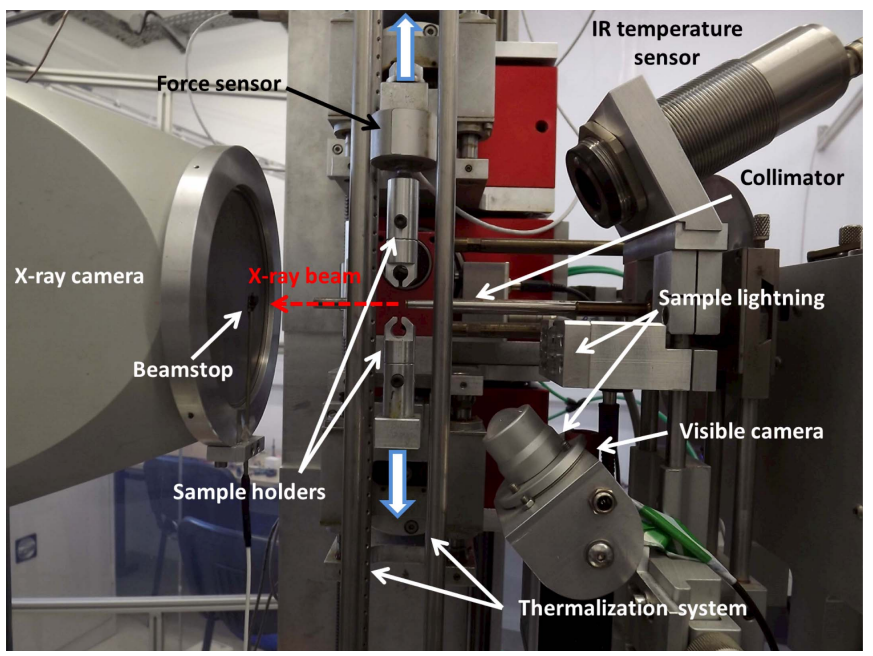

FIG. 2. Experimental setup for the XRD technique

The experimental setup used from the temperature measurement is presented in Figure 3. Tests were conducted with a

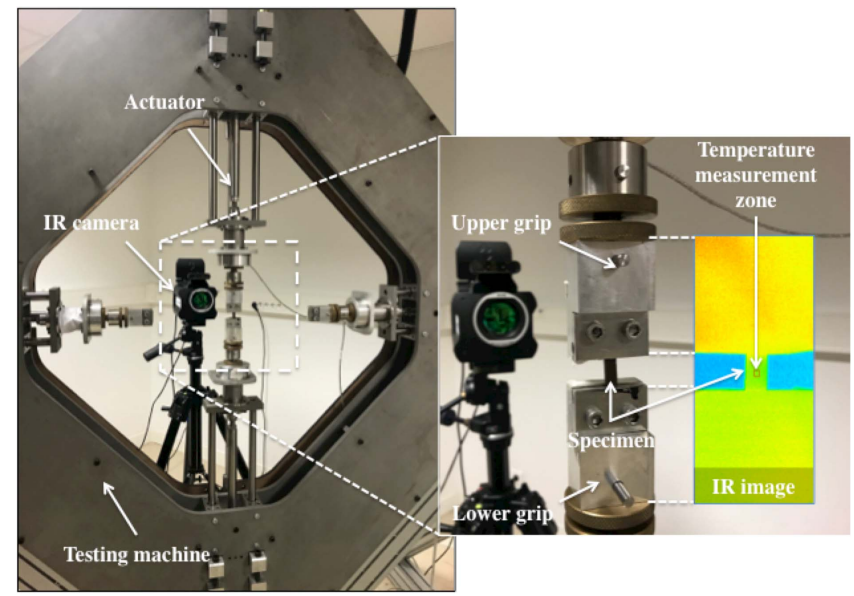

FIG. 3. Experimental setup for the temperature measurement

home-made biaxial testing machine. Four independent electrical actuators enables us to stretch symmetrically the specimens in two perpendicular directions. Actuators are driven by means of a home-made LabVIEW program. The cell load capacity is $1094 \mathrm{~N}$. In the present case, the testing machine is used to stretch symmetrically the specimen in one direction only. Therefore, the zone where the thermal measurement is performed remains in the 
same place, which enables us to obtain the temperature variation by subtracting the current measurement zone to the initial one, without any motion compensation technique ${ }^{25}$. In order to reduce the external radiation, the grips were covered with a black body leaf (see the IR image in Fig. 3).

\section{RESULTS}

Figure 4 presents the mechanical responses obtained in terms of the nominal stress, defined as the force per unit surface, in relation to the stretch for the two loading rates during the two types of measurement. The continuous and dotted lines are the
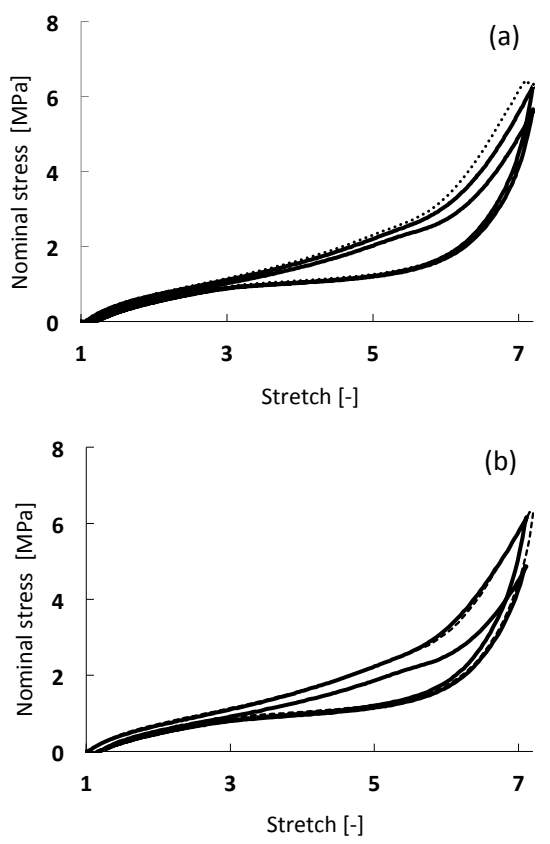

FIG. 4. Mechanical response obtained: XRD experiments in dotted line, IRT experiments in continuous line (a) $100 \mathrm{~mm} / \mathrm{min}$ (b) $200 \mathrm{~mm} / \mathrm{min}$

mechanical response obtained with the tensile machine used for IR thermography and XRD measurements, respectively.

The thermal response is shown in the red curves in Figure 5. This figure shows the typical response of a crystallizing rubber, i.e. a strong temperature increase occurs once the crystallization stretch onset is exceeded. It should noted that the temperature increases at the end of the unloading. This is due to non-adiabatic effects that are all the more significant that the loading rate is low. Further explanations on non-adiabatic effects on the temperature variation are provided in Ref. ${ }^{26}$ (pages 2721-2722).

The corresponding adiabatic temperature variations (curves in black color in Figure 5) are deduced from the heat source. The fact that $\tau(\lambda)$ enables us to retrieve a temperature equal to zero at the end of each cycle validates the characterization of the non-adiabatic effects, i.e. the value of $\tau$, as the material does not produce heat at each mechanical cycle.

Figure 6 depicts the heat source in relation to the stretch (curve A), obtained from Equation 3 (Step \#1). During loading, the heat source is positive and increases with the stretch. Once SIC starts, a strong increase in the heat produced is observed. The polynomial form in (Step \#2) is used to predict the heat source due to elastic couplings and to determine the area between the two curves, i.e.the thermal energy, due to SIC only. Then, the crystallization temperature is obtained from the primitive
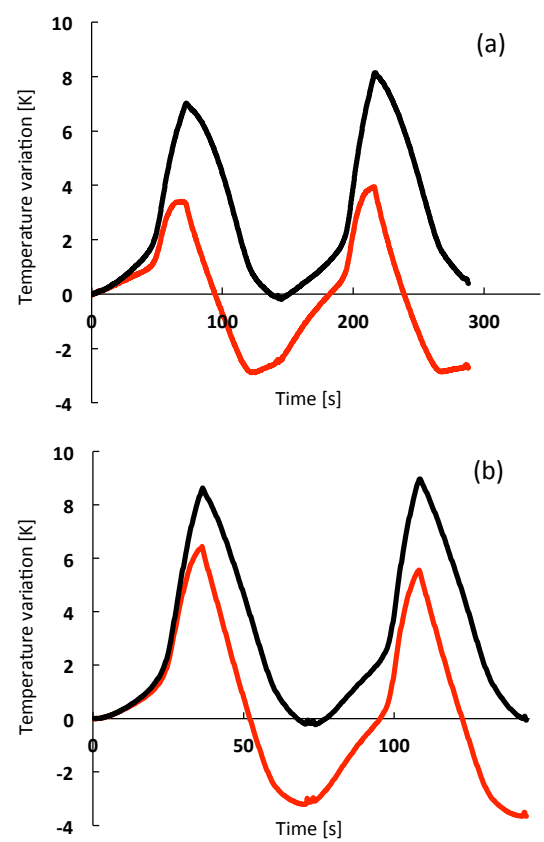

FIG. 5. Temperature variation measured (in red) and the corresponding adiabatic one (in black) during the first two cycles (a) $100 \mathrm{~mm} / \mathrm{min}$ (b) $200 \mathrm{~mm} / \mathrm{min}$
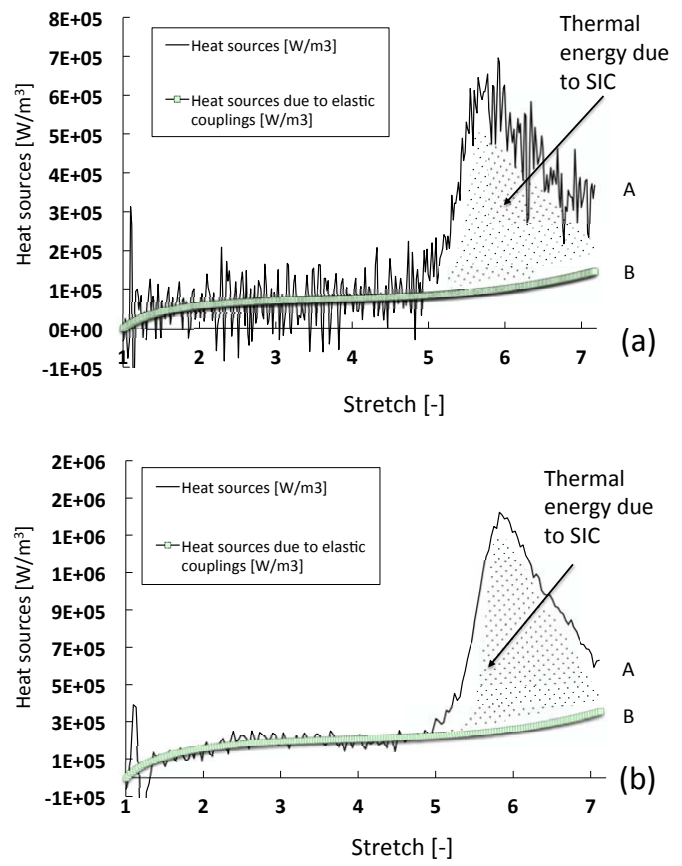

FIG. 6. Heat source, strain power density and heat source due to elastic couplings during the loading \#2 (a) $100 \mathrm{~mm} / \mathrm{min}$ (b) $200 \mathrm{~mm} / \mathrm{min}$

calculation of the heat source ${ }^{3}$ due to SIC.

The integration constant is determined considering that $T_{\text {cryst }}$ before crystallization starts is equal to zero. It should be noted that without any crystallization, i.e. below the strain at which crystallization starts during the loading, Tcryst is equal to zero, i.e. no temperature variation is due to SIC. Indeed, for this un-

\footnotetext{
${ }^{3}$ here the heat source is expressed in ${ }^{\circ} / \mathrm{s}$, i.e. S $/ \rho C$
} 
filled NR formulation the XRD measurements have shown that the crystallinity returns to zero at the end of each cycle. The crystallinity is calculated by using the fusion enthalpy. The crystallinity obtained for the two loading rates applied corresponds to continuous lines in Figure 7. The XRD measurement, which
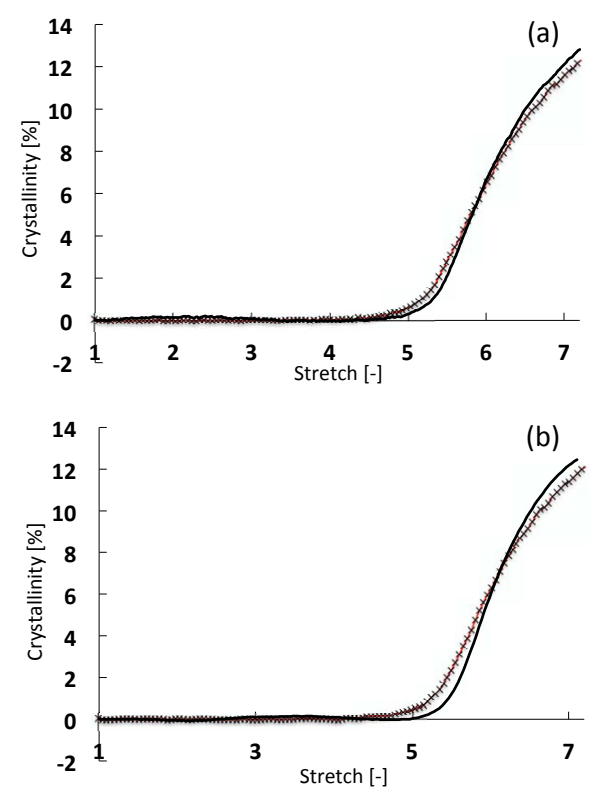

FIG. 7. Crystallinity in \% during the loading \#2 (-) surface calorimetry (x) XRD (a) $100 \mathrm{~mm} / \mathrm{min}$ (b) $200 \mathrm{~mm} / \mathrm{min}$

was performed during the first loading, corresponds to lines with cross symbol in the figure. This comparison clearly shows the relevancy of evaluating strain-induced crystallization from IR thermography measurements.

\section{CONCLUSION}

In the present study, the crystallinity of an unfilled natural rubber has been evaluated by two different techniques: the IR thermography based quantitative surface calorimetry (IRT-QSC) and the $\mathrm{X}$-ray diffraction (XRD) techniques. Results obtained highlight a very satisfactory agreement between the two measurements, which validates the IR-QSC technique proposed in Le Cam (2018) to measure the strain-induced crystallinity. Further investigations are currently carried out by coupling the two techniques for a better characterization of the thermo-physical properties and their evolution with the stretch. It is to note that X-ray diffraction is also complementary of temperature measurements at low drawing velocity where calorific effects become hardly detectable.

\section{AUTHOR'S CONTRIBUTIONS}

All authors contributed equally to this work.

\section{DATA AVAILABILITY STATEMENT}

The data that support the findings of this study are available from the corresponding author upon reasonable request.

[1]J. Katz, "Rntgenspektrographische untersuchungen am gedehnten kautschuk und ihre mgliche bedeutung fr das problem der dehnungseigenschaften dieser substanz," Naturwiss 4, 169 (1925)

[2]C. Bunn, "Bakerian lecture - the physical interpretation of quantum mechanics," Proceedings of the Royal Society A 180, 40 (1942)
[3]Y. Takahashi and T. Kumano, "Crystal structure of natural rubber,” Macromolecules 37, 4860 (2004)

[4]A. Immirzi, C. Tedesco, G. Monaco, and A. Tonelli, "Crystal structure and melting entropy of natural rubber," Macromolecules 38, 1223 (2005)

[5]G. Rajkumar, J. Squire, and S. Arnott, "A new structure for crystalline natural rubber,", Macromolecules 39, 7004 (2006)

[6]S. Toki, I. Sics, S. Ran, L. Liu, B. Hsiao, S. Murakami, M. Tosaka, S. Kohjiya, S. Poompradub, Y. Ikeda, and A. Tsou, "Structural developments in synthetic rubbers during uniaxial deformation by in situ synchrotron x-ray diffraction," Journal of Polymer Science Part B: Polymer Physics 42, 956-964 (2004)

[7]S. Toki, T. Fujimaki, and M. Okuyama, "Strain-induced crystallization of natural rubber as detected real-time by wide-angle $\mathrm{x}$-ray diffraction technique," Polymer 41, 5423-5429 (2000)

[8]S. Trabelsi, P.-A. Albouy, and J. Rault, "Effective local deformation in stretched filled rubber," Macromolecules 36, 9093-9099 (2003)

[9]D. Göritz and F. Müller, "Die kalorimetrische erfassung der dehnungskristallisation polymerer," Kolloid-Zeitschrift und Zeitschrift für Polymere 241, 10751079 (1970)

[10]S. Trabelsi, P.-A. Albouy, and J. Rault, "Stress-induced crystallization around a crack tip in natural rubber," Macromolecules 35, 10054-10061 (2002)

[11]P. Rublon, B. Huneau, E. Verron, N. Saintier, S. Beurrot, A. Leygue, C. Mocuta, D. Thiaudière, and D. Berghezan, "Multiaxial deformation and straininduced crystallization around a fatigue crack in natural rubber," Engineering Fracture Mechanics 123, 59-69 (2014)

[12]A. Chrysochoos, "Analyse du comportement des matériaux par thermographie infra rouge," in Colloque Photomécanique, Vol. 95 (1995) pp. 201-211

[13]J. Samaca Martinez, J.-B. Le Cam, X. Balandraud, E. Toussaint, and J. Caillard, "Mechanisms of deformation in crystallizable natural rubber. part 2: Quantitative calorimetric analysis," Polymer 54, 2727-2736 (2013)

[14]J.-B. Le Cam, J. Samaca Martinez, X. Balandraud, E. Toussaint, and J. Caillard, "Thermomechanical analysis of the singular behavior of rubber: Entropic elasticity, reinforcement by fillers, strain-induced crystallization and the mullins effect," Experimental Mechanics 55, 771-782 (2015)

[15]J. Samaca Martinez, X. Balandraud, E. Toussaint, J.-B. Le Cam, and D. Berghezan, "Thermomechanical analysis of the crack tip zone in stretched crystallizable natural rubber by using infrared thermography and digital image correlation," Polymer 55, 6345-6353 (2014)

[16]J. R. Samaca Martinez, E. Toussaint, X. Balandraud, J.-B. Le Cam, and D. Berghezan, "Heat and strain measurements at the crack tip of filled rubber under cyclic loadings using full-field techniques," Mechanics of Materials 81, 62-71 (2015)

[17]J.-B. Le Cam, "Energy storage due to strain-induced crystallization in natural rubber: the physical origin of the mechanical hysteresis," Polymer 127, 166173 (2017)

[18]A. Lachhab, E. Robin, J.-B. Le Cam, F. Mortier, Y. Tirel, and F. Canevet, "Energy stored during deformation of crystallizing tpu foams," Strain e12271 (2018), 10.1111/str.12271

[19]M. Loukil, G. Corvec, E. Robin, M. Miroir, J.-B. Le Cam, and P. Garnier, "Stored energy accompanying cyclic deformation of filled rubber," European Polymer Journal 98, 448-455 (2018)

[20]J.-B. Le Cam, "Strain-induced crystallization in rubber: A new measurement technique," Strain 54, e12256 (2018)

[21]P.-A. Albouy, A. Vieyres, R. Perez-Aparicio, O. Sanseau, and P. Sotta, "The impact of strain-induced crystallization on strain during mechanical cycling of cross-linked natural rubber," Polymer 55, 4022-4031 (2014)

[22]P.-A. Albouy and P. Sotta, "Draw ratio at the onset of strain-induced crystallization in cross-linked natural rubber," Macromolecules 53, 992-1000 (2020)

[23]T. Spratte, J. Plagge, M. Wunde, and M. Klppel, "Investigation of straininduced crystallization of carbon black and silica filled natural rubber composites based on mechanical and temperature measurements," Polymer 115, 12-20 (2007)

[24]L. Treloar, "The elasticity and related properties of rubbers," Reports on Progress in Physics 36, 755 (1973)

[25]E. Toussaint, X. Balandraud, J.-B. Le Cam, and M. Grediac, "Combining displacement, strain, temperature and heat source fields to investigate the thermomechanical response of an elastomeric specimen subjected to large deformations," Polymer Testing 31, 916-925 (2012)

[26]J. Samaca Martinez, J.-B. Le Cam, X. Balandraud, E. Toussaint, and J. Caillard, "Mechanisms of deformation in crystallizable natural rubber. part 1: Thermal characterization," Polymer 54, 2717-2726 (2013) 